\title{
Extreme Energy Events Project: construction of the detectors
}

\author{
F. Carnesecchi; ${ }^{11,6}$, M.Abbrescia ${ }^{1,2}$, C.Avanzini ${ }^{1,3}$, L.Baldini ${ }^{1,3}$, R.Baldini Ferroli ${ }^{1,4}$, \\ G.Batignani $^{1,3}$, M.Battaglieri ${ }^{1,17}$, S.Boi ${ }^{1,8}$, E.Bossini ${ }^{1,5}$, A.Chiavassa ${ }^{1,7}$, C.Cicalo ${ }^{1,8}$, L. \\ Cifarelli $^{1,6}$, F.Coccetti ${ }^{1}$, E.Coccia ${ }^{1,9}$, A.Corvaglia ${ }^{1,10}$, D.De Gruttola ${ }^{1}$, S. De \\ Pasquale $^{1,11}$, F.L.Fabbri ${ }^{1,4}$, V.Frolov ${ }^{16}$, P.Galeotti ${ }^{1,7}$, M.Garbini ${ }^{1,6}$, G.Gemme ${ }^{1,17}$, \\ I.Gnesi $^{1,7}$, S.Grazzi ${ }^{1}$, C.Gustavino ${ }^{1,12}$, D.Hatzifotiadou ${ }^{1,6,15}$, P.LaRocca ${ }^{1,18}$, \\ G.Mandaglio $^{1,19}$, O.Maragoto Rodriguez ${ }^{14}$, G.Maron ${ }^{13}$, M.N.Mazziotta ${ }^{1,20}$, S.Miozzi ${ }^{1,4}$, \\ R.Nania ${ }^{1,6}$, F.Noferini ${ }^{1,6}$, F.Nozzoli ${ }^{1,21}$, F.Palmonari ${ }^{1,6}$, M.Panareo ${ }^{1,10}$, M.P.Panetta ${ }^{1,10}$, \\ R.Paoletti ${ }^{1,5}$, W.Park ${ }^{14}$, L.Perasso ${ }^{1,17}$, F.Pilo ${ }^{1,3}$, G.Piragino ${ }^{1,7}$, S.Pisano ${ }^{1,4}$, F.Riggi ${ }^{1,18}$, \\ G.C.Righini $^{1}$, C.Ripoli ${ }^{1,11}$, M.Rizzi ${ }^{1,2}$, G.Sartorelli ${ }^{1,6}$, E.Scapparone ${ }^{1,6}$, \\ M.Schioppa ${ }^{1,22}$, A.Scribano ${ }^{1,3}$, M.Selvi ${ }^{1,6}$, S.Serci ${ }^{1,8}$, S.Squarcia ${ }^{1,17}$, M.Taiuti ${ }^{1,17}$, \\ G.Terreni $^{1,3}$, A.Trifirò ${ }^{1,23}$, M.Trimarchi ${ }^{1,23}$, M.C.Vistoli $^{13}$, L.Votano ${ }^{1,12}$, \\ M.C.S.Williams ${ }^{1,6,15}$, L.Zheng ${ }^{1,14,15}$, A.Zichichi ${ }^{1,6,15}$, R.Zuyeuski ${ }^{1,14,15}$ \\ ${ }^{1}$ Museo Storico della Fisica e Centro Studi e Ricerche Enrico Fermi, Roma, Italy \\ ${ }^{2}$ INFN and Dipartimento Interateneo di Fisica, Università di Bari, Bari, Italy \\ ${ }^{3}$ INFN and Dipartimento di Fisica, Università di Pisa, Pisa, Italy \\ ${ }^{4}$ INFN, Laboratori Nazionali di Frascati, Frascati (RM), Italy \\ ${ }^{5}$ INFN Gruppo Collegato di Siena and Dipartimento di Fisica, Università di Siena, Siena, Italy \\ ${ }^{6}$ INFN and Dipartimento di Fisica e Astronomia, Università di Bologna, Bologna, Italy \\ ${ }^{7}$ INFN and Dipartimento di Fisica, Università di Torino, Torino, Italy \\ ${ }^{8}$ INFN and Dipartimento di Fisica,Università di Cagliari, Cagliari, Italy \\ ${ }^{9}$ INFN and Dipartimento di Fisica, Università di Roma Tor Vergata, Roma, Italy \\ ${ }^{10}$ INFN and Dipartimento di Matematica e Fisica, Università del Salento, Lecce, Italy \\ ${ }^{11}$ INFN and Dipartimento di Fisica, Università di Salerno, Salerno, Italy \\ 12 INFN, Laboratori Nazionali del Gran Sasso, Assergi (AQ), Italy \\ ${ }^{13}$ INFN CNAF, Bologna, Italy \\ ${ }^{14}$ ICSC World Laboratory, Geneva, Switzerland \\ ${ }^{15}$ CERN, Geneva, Switzerland \\ 16 JINR Joint Institute for Nuclear Research, Dubna, Russia \\ ${ }^{17}$ INFN and Dipartimento di Fisica, Università di Genova, Genova, Italy \\ ${ }^{18}$ INFN and Dipartimento di Fisica e Astronomia, Università di Catania, Catania, Italy \\ ${ }^{19}$ INFN Sezione di Catania and Dipartimento di Scienze Chimiche, Biologiche, Farmaceutiche e \\ Ambientali, Università di Messina, Messina, Italy \\ ${ }^{20}$ INFN Sezione di Bari, Bari, Italy \\ ${ }^{21}$ INFN and ASI Science Data Center, Roma, Italy \\ ${ }^{22}$ INFN and Dipartimento di Fisica, Università della Calabria, Cosenza, Italy \\ ${ }^{23}$ INFN Sezione di Catania and Dipartimento di Scienze Matematiche e Informatiche, Scienze \\ Fisiche e Scienze della Terra, Università di Messina, Messina, Italy \\ E-mail: francesca.carnesecchi@cern.ch
}


The Extreme Energy Events (EEE) experiment is a strategic project of Centro Fermi dedicated to the study of extreme energy cosmic rays with an innovative outreach approach: high school students are directly involved in the experiment and play a primary role. EEE exploits a network of muon tracking telescopes constituted by three large area MRPCs. In this work we describe the detector design and the construction phase which is performed at CERN by high school students and teachers. The experiment is coordinated by Centro Fermi with collaboration from INFN, CERN, Ministero dell'Università e della Ricerca (MIUR). Nowadays 52 telescopes are installed all over Italy $\left(310^{5} \mathrm{~km}^{2}\right)$ and a total of 100 institutes participate monitoring the quality of the data and analysing them. The EEE Project is still expanding to enlarge the network, involving more schools.

The European Physical Society Conference on High Energy Physics 5-12 July, 2017

Venice

* Speaker. 


\section{Introduction}

The Extreme Energy Events Project [1] [2] is a very large array of particle detectors distributed all over Italian territory and located inside High Schools buildings and INFN Sections and Laboratories. The experiment aim is to study cosmic rays by detecting and tracking the muon component of the Extensive Air Showers (EAS) produced by the interaction of high energy primary cosmic rays in the Earth atmosphere. The detector is a GPS synchronised tracking telescope made of three large area Multigap Resistive Plate Chambers (MRPC); these detectors are used in many experiments due to their excellent efficiency and time resolution [3].

The chambers are built and tested by students at CERN and then installed to assemble the telescopes inside high school institutes. The students then operate and monitor the telescopes during the data acquisition and they take part in the data analysis. The project started in 2004 and since then 52 telescopes have been built and installed in the schools; the network is still expanding.

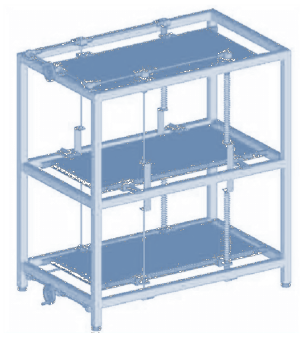

(a)

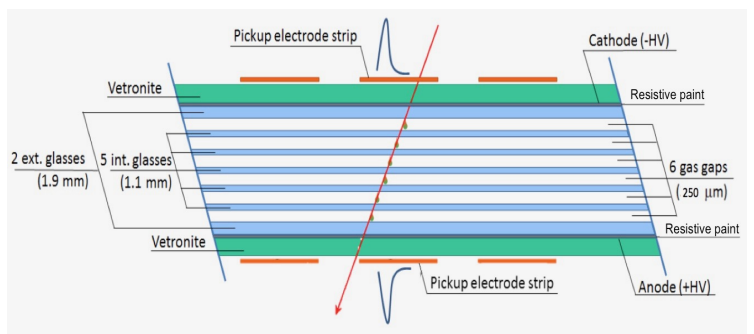

(b)

Figure 1: EEE Project telescope drawing (a) and EEE MRPC basic design of the (b).

\section{Detector Design and construction}

The EEE Project telescope is composed by 3 MRPCs as shown in Fig.1(a). These MRPCs are a wider and cheaper version of the Time-Of-Flight (TOF) detector of the ALICE experiment at LHC [3].

Each MRPC $\left(0.8 \times 1.6 \mathrm{~m}^{2}\right.$ active area), is a stack of resistive plates. It works in a streamer-free operation mode. The basic design of the MRPC , Figure 1(b), consists of six gas gaps of $250 \mu \mathrm{m}$, filled with a $98 \% \mathrm{C}_{2} \mathrm{~F}_{4} \mathrm{H}_{2}$ and $2 \% \mathrm{SF}_{6}$ gas mixture. The resistive plates are commercially available soda lime glass with a thickness of $1.9 \mathrm{~mm}$ for the outer glass sheets and $1.1 \mathrm{~mm}$ for the inner sheets. The external glass sheets are coated with a resistive paint to create a resistive electrode with a surface resistivity of about $5 M \Omega /$ square.

The high voltage to the MRPCs is applied by means of DC/DC converters which supply up to $\pm 10 \mathrm{kV}$ when powered with 0 to $5 \mathrm{~V}$. The signal initiated by a charged particle traversing the detector is formed on the 24 readout copper strips; these are obtained by applying copper tape ( $2.5 \mathrm{~cm}$ wide) on vetronite panels. The signal then drifts to both ends of the MRPC where they are read by front-end cards ( 24 channels each) provided with an ultra-fast and low power amplifier/discriminator designed for MRPC operation: the NINO ASIC chip[4]. A total of 144 channels is then used for each telescope and time measurements are performed using commercial multi-hit TDCs. Two honeycomb panels ( $15 \mathrm{~mm}$ thick) reinforce the structure which is then enclosed in an 
aluminum box provided with standard gas connectors.

The detectors are built by teams of high school students and teachers at CERN, under the supervision of expert researchers. The students experience how it is possible to build a cutting-edge detector with excellent performance, starting from simple materials (glass, plastic etc). Pictures of two phases of the construction are shown in Figure 2: the readout electrodes (strips) Figure 2(a) and the $250 \mu \mathrm{m}$ thick gaps between the glass sheets, obtained by using a commercial fishing line run across the surface, Figure 2(b).

The construction shift lasts for 5 days; during this period the team builds the three detectors

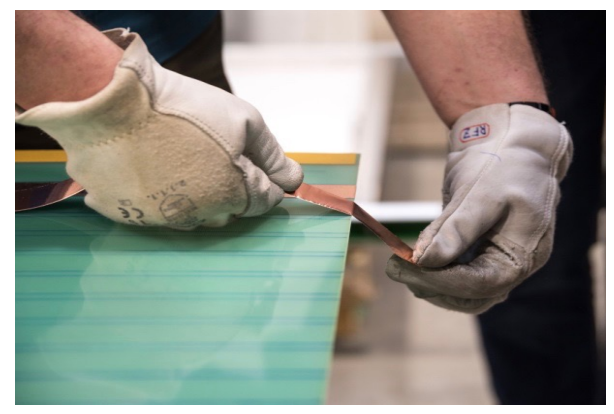

(a)

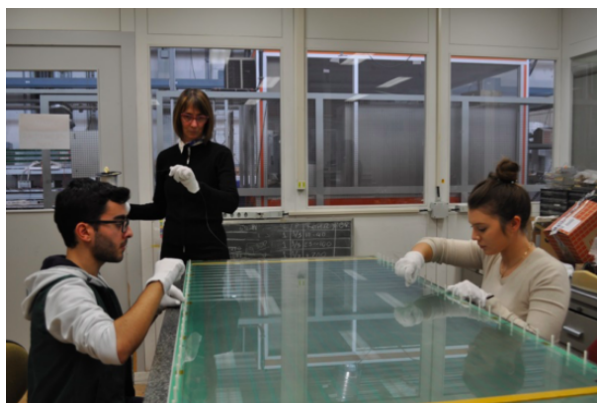

(b)

Figure 2: Readout electrodes construction by application of copper tape on the vetronite panel (a). The $250 \mu \mathrm{m}$ diameter fishing line (used as spacer between glass sheets) stretched across the surface of the glass from one side to the other and around plastic screws fixed at both sides of the chamber (b).

needed for their school telescope. After construction the MRPCs are delivered to the school and the telescope is installed. Students and teachers participate also to the installation phase. Moreover after the installation, students are in charge of monitoring the telescope and they also contribute to data analysis. This approach guarantees that Italian young students play a primary role in the EEE Project experiment and thanks to their contribution long term data taking runs have been performed, providing data for physics analysis.

\section{Detector Performance}

Until today more than 150 MRPCs have been built by teams of students. After construction all the detectors are tested to check their performances, starting from the measurement of the detection efficiency. All the built detectors show efficiency close to 100\% at applied voltage of $18 \mathrm{kV}$. An example of efficiency curve, obtained with cosmic rays, as a function of the applied voltage is shown in Figure 3(a) for three chambers built by students from Siena, Italy. Figure 3(b) and Figure 4 respectively show the time and spatial resolution obtained with comic rays. A spatial resolution of the order of $1 \mathrm{~cm}^{2}$ is obtained, and the time resolution is in the order of $240 \mathrm{ps}$. In a test beam [5], with same structure of MRPC a time resolution of $70 \mathrm{ps}$ has been reached ${ }^{1}$.

\footnotetext{
${ }^{1}$ The different time resolution is due to the experimental conditions in cosmic rays and beam test (i.e. beam spot and particle directions).
} 


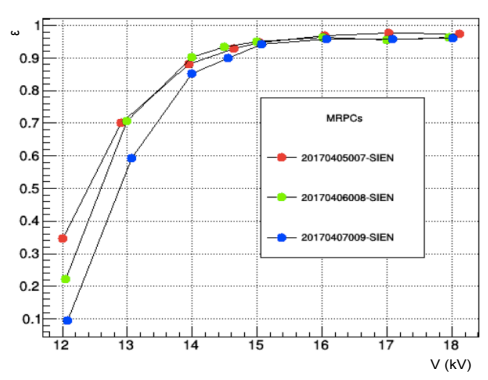

(a)

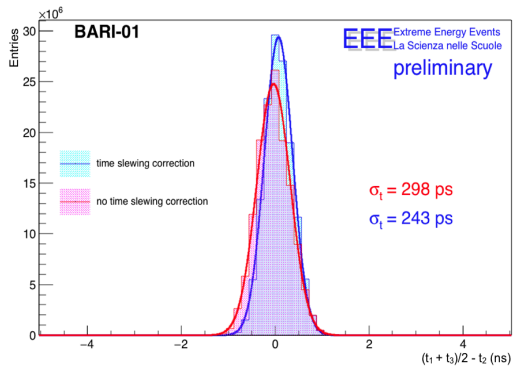

(b)

Figure 3: Efficiency as a function of applied HV for three MRPCs. (a). Time resolution as a function of applied HV of MRPC before and after time slewing corrections (b).

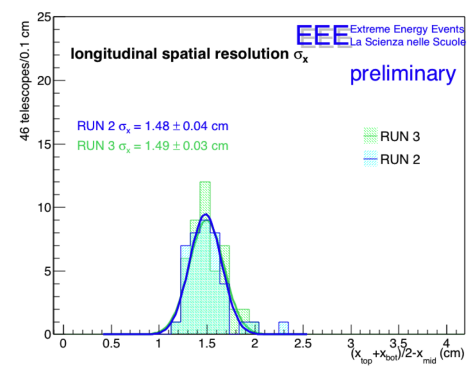

(a)

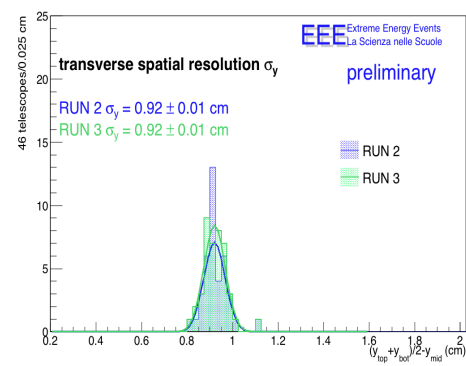

(b)

Figure 4: Longitudinal (a) and transverse (b) spatial resolution.

\section{Conclusions}

The Extreme Energy Events Project experiment is dedicated to the study of extreme energy cosmic rays with an innovative outreach approach: high school students are directly involved in the experiment and they play a primary role starting from the detector construction. More than 50 telescopes have been built by students and installed in high schools distributed over the whole of Italy. The EEE Project is still expanding with the aim of enlarging the network and involving more schools in the EEE network.

\section{References}

[1] A. Zichichi, Progetto "La Scienza nelle Scuole" EEE-Extreme Energy Events, Italian Physical Society (2005).

[2] http://eee.centrofermi.it/

[3] A. Akindinov et al., Nucl. Instr. and Meth. A 456 (2000) 16.

[4] F. Anghinolfi et al.,Nucl. Instr. and Meth. A 533 (2004) 183.

[5] M. Abbrescia et al.,Nucl. Instr. and Meth. A 593 (2008) 263. 